\title{
Camouflage treatment of posterior bite collapse in a patient with skeletal asymmetry by using posterior maxillary segmental osteotomy
}

\author{
Haitham Badra (1) \\ Soo-Yeon Lee ${ }^{\mathrm{b}}$ \\ Hong-Sik Park ${ }^{\mathrm{c}}$ \\ Joo-Young Ohe ${ }^{\mathrm{d}}$ \\ Yoon-Goo Kang ${ }^{\mathrm{e}}$ \\ Hyo-Won Ahn ${ }^{\mathrm{e}}$ (1)
}

${ }^{a}$ Department of Orthodontics, Kyung Hee University Dental Hospital, Seoul, Korea

${ }^{\mathrm{b}}$ Department of Dentistry, Graduate School, Kyung Hee University, Seoul, Korea

'Private Practice, Seoul, Korea

${ }^{d}$ Department of Oral \& Maxillofacial Surgery, Kyung Hee University School of Dentistry, Seoul, Korea

'Department of Orthodontics, Kyung Hee University School of Dentistry, Seoul, Korea
Orthodontic treatment of posterior bite collapse due to early loss of molars and the consequent drift of adjacent teeth is complicated. When the posterior bite collapse occurs in patients with facial asymmetry, both transverse and vertical compensation are necessary for camouflage orthodontic treatment. In such cases, posterior maxillary segmental osteotomy (PMSO) can be an effective alternative procedure that simplifies the orthodontic treatment and shows long-term stability through dental compensation within the alveolar bone housing. This case report aimed to describe the orthodontic treatment of maxillary occlusal plane canting caused by severely extruded maxillary teeth in a patient with skeletal facial asymmetry that was corrected with PMSO along with protraction of the lower second molar to replace the space of the extracted first molar. The treatment duration was 18 months, and stable results were obtained after 2 years of retention.

[Korean J Orthod 2020;50(4):278-289]

Key words: Posterior segmental osteotomy, Bite collapse, Facial asymmetry

Received January 8, 2019; Revised March 14, 2019; Accepted March 29, 2019.

Corresponding author: Hyo-Won Ahn.

Associate Professor, Department of Orthodontics, Kyung Hee University School of Dentistry, 26 Kyungheedae-ro, Dongdaemun-gu, Seoul 02447, Korea.

Tel +82-2-958-9390 e-mail hyowon@khu.ac.kr

How to cite this article: Badr H, Lee SY, Park HS, Ohe JY, Kang YG, Ahn HW. Camouflage treatment of posterior bite collapse in a patient with skeletal asymmetry by using posterior maxillary segmental osteotomy. Korean J Orthod 2020;50:278-289.

(C) 2020 The Korean Association of Orthodontists.

This is an Open Access article distributed under the terms of the Creative Commons Attribution Non-Commercial License (http://creativecommons.org/licenses/by-nc/4.0) which permits unrestricted non-commercial use, distribution, and reproduction in any medium, provided the original work is properly cited. 


\section{INTRODUCTION}

Prolonged tooth loss without replacement may disrupt masticatory function or cause temporomandibular joint (TMJ) disorders, deviation of mandibular movement, and supraeruption of opposing teeth, leading to a loss in vertical dimension called posterior bite collapse.' Generally, as the number of affected teeth increases, the extent of posterior bite collapse becomes more severe. The loss of posterior occlusal support causes repositioning of the mandible, complicating rehabilitation of the opposing teeth. ${ }^{1,2}$

Occlusal plane canting is a distinct feature of posterior bite collapse. If the mandibular molars are missing, the opposing maxillary molars would extrude, while the mandibular teeth would tilt and migrate toward the extracted site. Thus, in patients with facial asymmetry, treatment of posterior bite collapse would be even more complex due to the vertical and transverse dental compensation. ${ }^{3}$ In such patients, transverse compensation should be considered simultaneously while correcting the vertical dimension.

The combination of surgery and orthodontic treatment results in good functional and esthetic outcomes with long-term stability. However, if orthognathic surgery is rejected by patients with facial asymmetry and posterior bite collapse, then posterior maxillary segmental osteotomy (PMSO) can be performed as an alternative. This report presents a case showing effective vertical and transverse control by using PMSO in the treatment of posterior bite collapse in a patient with facial asymmetry.

\section{DIAGNOSIS AND ETIOLOGY}

A 22-year-old female patient visited Department of Orthodontics, Kyung Hee University Dental Hospital with chief complaints of difficulty in chewing on the left side and an asymmetric appearance. Clinical examination revealed chin deviation to the right side with lip-line canting (higher right side) and an incompetent lip. The patient showed severe dentoalveolar extrusion of the maxillary left posterior teeth due to a retained root of the opposing mandibular first molar and mesially tilted second and third molars. The mandibular left premolars were also extruded and inclined lingually, which resulted in a scissors-bite on the left premolar area. A localized crossbite was observed between the maxillary right canine and mandibular right canine and first premolar. The upper dental midline coincided with the facial midline, but the lower dental midline deviated $3.0 \mathrm{~mm}$ to the right. The canine relationship revealed Class 11 occlusion on the right side and Class 111 on the left, and mild anterior crowding in both arches (Figures 1 and 2).

The cephalometric analysis showed a skeletal Class 1 relationship (ANB, $2.9^{\circ}$ ), a hyperdivergent vertical pattern (SUM angle, $400.4^{\circ}$; PFH/AFH, 59.0\%; FMA, 30.5 ), and normal inclination of the maxillary and mandibular incisors (U1-FH, $116.6^{\circ}$; IMPA, 88.0 $)$. Cone-beam computed tomography (CBCT) analysis showed facial

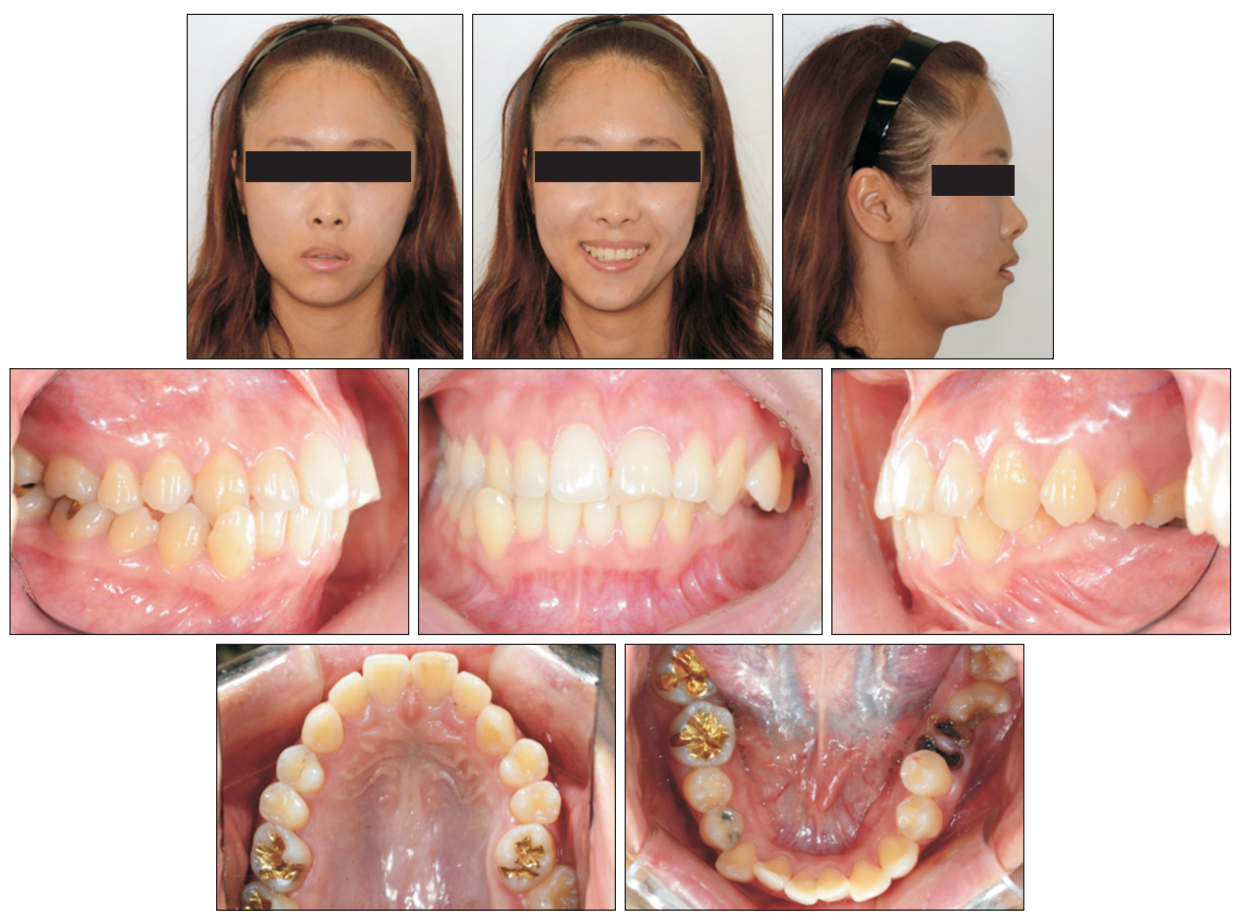

Figure 1. Pretreatment facial and intraoral photographs. 

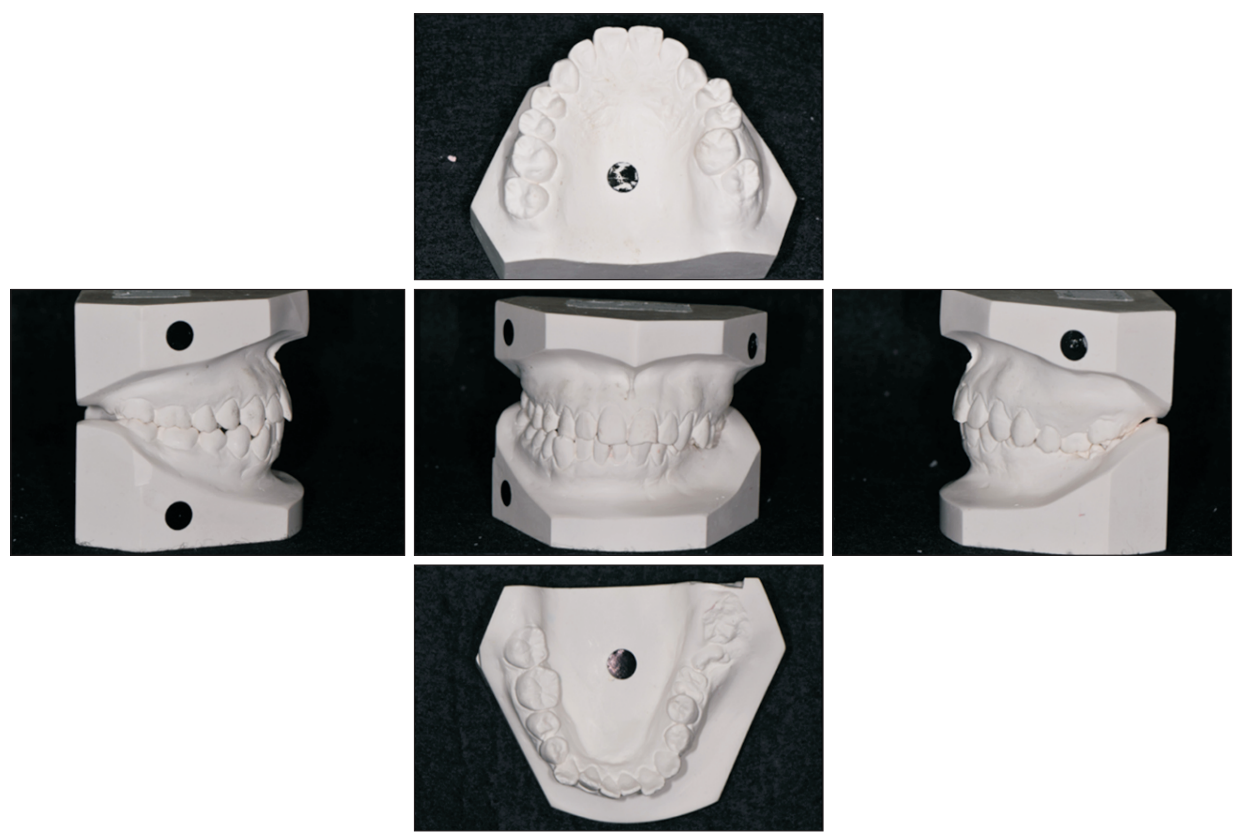

Figure 2. Pretreatment cast models.
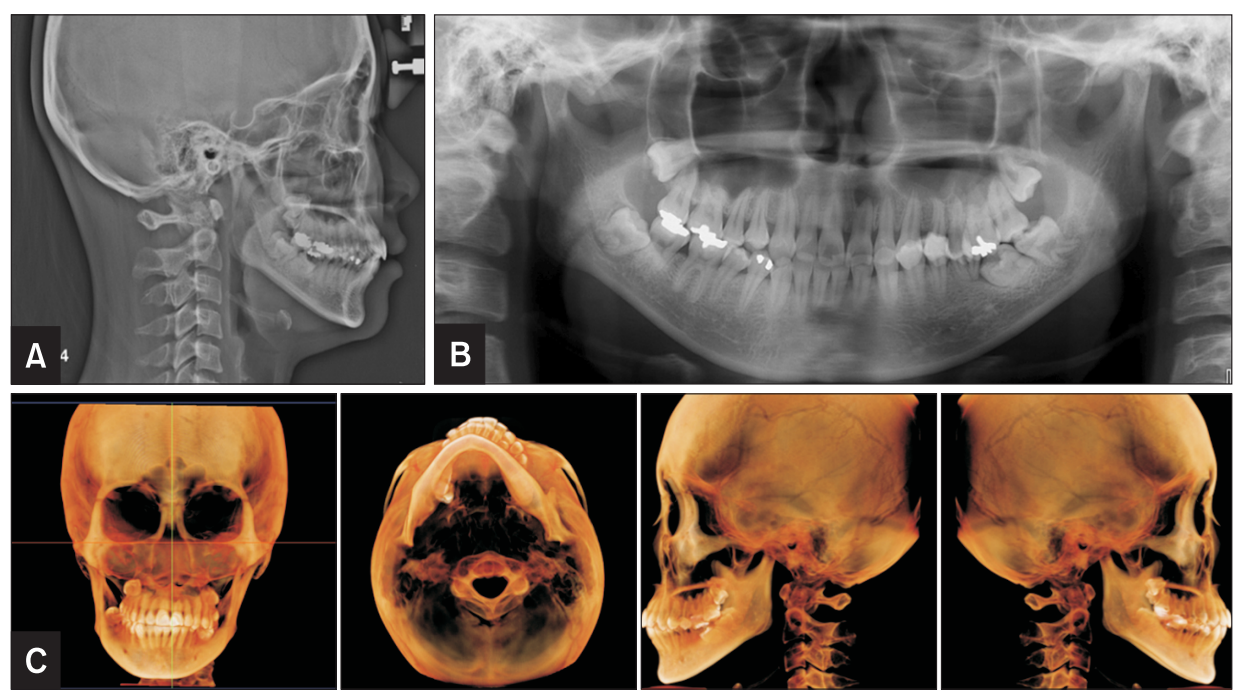

Figure 3. Pretreatment radiographs. A, Lateral cephalogram; B, panoramic radiograph; and $\mathbf{C}$, cone-beam computed tomography images.

asymmetry with translation-dominant type (T-type). The characteristics of T-type asymmetry include discrepancy of frontal ramal inclination between the left and right sides with menton deviation with no significant ramal height discrepancy. ${ }^{4}$ Although the patient showed an occlusal plane cant, it originated from dental bite collapse rather than a maxillary skeletal cant. The menton had deviated $5.5 \mathrm{~mm}$ to the right side. The maxillary left posterior alveolus was elongated and the occlusal plane was canted with the right side up by $3.0 \mathrm{~mm}$ and 5.5 $\mathrm{mm}$ at the canine and first molar levels, respectively. The panoramic radiograph revealed periapical radiolucency of the maxillary left central incisor, impacted wisdom teeth, and residual root of the mandibular left first molar (Fig- ure 3 and Table 1).

Although the radiograph showed degenerative condylar changes, there were no TMJ symptoms, and the patient was referred to a TMJ specialist for regular check-ups during the orthodontic treatment. Root canal treatment of the left maxillary central incisor was performed, and bracket bonding was delayed until bone healing was confirmed on the radiograph.

Informed consent of the patient was obtained for release of the patient's records.

\section{TREATMENT OBJECTIVES}

The treatment objective was to correct the posterior 
Badr et al • Posterior maxillary segmental osteotomy for posterior bite collapse

Table 1. Cephalometric measurements at pretreatment, post-treatment, and post-retention stages

\begin{tabular}{|c|c|c|c|c|}
\hline Measurement & Korean female norm & Pretreatment & Posttreatment & Postretention \\
\hline \multicolumn{5}{|l|}{ Skeletal (anteroposterior) } \\
\hline SNA $\left({ }^{\circ}\right)$ & 81.6 & 83.0 & 83.1 & 82.9 \\
\hline N perp-point A (mm) & 0.9 & 2.9 & 2.9 & 2.8 \\
\hline $\operatorname{SNB}\left({ }^{\circ}\right)$ & 79.2 & 80.0 & 80.1 & 80.0 \\
\hline N perp-Pog $(\mathrm{mm})$ & -2.0 & -1.8 & -1.8 & -1.8 \\
\hline $\operatorname{ANB}\left({ }^{\circ}\right)$ & 2.5 & 2.9 & 2.9 & 2.9 \\
\hline \multicolumn{5}{|l|}{ Skeletal (vertical) } \\
\hline $\operatorname{SUM}\left({ }^{\circ}\right)$ & 393.3 & 400.4 & 400.0 & 399.7 \\
\hline $\mathrm{PFH} / \mathrm{AFH}(\%)$ & 66.8 & 59.0 & 59.6 & 59.3 \\
\hline $\mathrm{OP}-\mathrm{FH}\left({ }^{\circ}\right)$ & 8.8 & 3.0 & 10.4 & 10.0 \\
\hline $\mathrm{MP}-\mathrm{FH}\left({ }^{\circ}\right)$ & 24.3 & 30.5 & 30.8 & 30.6 \\
\hline \multicolumn{5}{|l|}{ Dento-alveolar } \\
\hline Interincisal angle $\left(^{\circ}\right)$ & 123.8 & 124.6 & 126.6 & 126.2 \\
\hline $\mathrm{U} 1-\mathrm{FH}\left({ }^{\circ}\right)$ & 116.0 & 116.6 & 112.7 & 112.0 \\
\hline $\operatorname{IMPA}\left({ }^{\circ}\right)$ & 95.9 & 88.0 & 89.7 & 90.7 \\
\hline U6-palatal plane $(\mathrm{mm})$ & 24.8 & 29.0 & 24.0 & 23.8 \\
\hline U1-palatal plane $(\mathrm{mm})$ & 28.5 & 31.0 & 32.0 & 32.5 \\
\hline U1 exposure (mm) & 2.0 & 2.6 & 3.2 & 3.0 \\
\hline L6-MP (mm) & 34.0 & 24.0 & 31.0 & 32.0 \\
\hline L1-MP (mm) & 42.6 & 44.2 & 43.0 & 43.0 \\
\hline \multicolumn{5}{|l|}{ Soft tissue } \\
\hline Upper lip to E-Plane & -0.9 & 0.0 & 0.2 & 0.2 \\
\hline Lower lip to E-Plane & 0.6 & 1.0 & 2.0 & 2.3 \\
\hline
\end{tabular}

SNA, Sella-Nasion to A Point angle; N perp-point A, distance from point A to perpendicular line from Nasion; SNB, sellaNasion to B Point angle; N perp-Pog, distance from Pogonion to perpendicular line from Nasion; ANB, A point-Nasion to B point angle; SUM, sum of saddle angle, articular angle, and gonial angle; $\mathrm{PFH} / \mathrm{AFH}$, ratio of posterior facial height to anterior facial height; OP-FH angle, angle between occlusal plane and Frankfort (FH) plane; MP-FH angle, angle between FH plane and mandibular plane (MP, the line connecting Gonion-Menton); U1-FH, upper incisor to FH plane angle; IMPA, lower incisor to MP angle; U6-palatal plane, distance from upper first molar to palatal plane; U1-palatal plane, distance from upper incisor to palatal plane; U1 exposure, distance from upper lip (Stomion) to incisor tip; L6-MP, distance from lower first molar to MP; L1-MP, distance from lower incisor to MP; Upper lip E-line, distance from upper lip to E-line; Lower lip E-line, distance from lower lip to E-line.

bite collapse and establish balanced occlusal function by performing the following steps: (1) maxilla, repositioning of the left elongated posterior segment to attain vertical space for rehabilitation of the opposing mandibular teeth; (2) mandible, uprighting and protracting the mesially tilted mandibular left second and third molars into the first molar area; and (3) correction of the crossbite on the right side and midline discrepancy.

\section{TREATMENT ALTERNATIVES}

The treatment options vary depending on the amount of vertical and transverse discrepancy. For the verti- cal aspect, it is possible to regain the interarch space with coronoplasty following endodontic treatment of supraerupted teeth in mild to moderate cases. With the increasing use of temporary anchorage devices (TADs) in orthodontics, molar intrusion and occlusal plane canting correction with minimal surgical intervention has been reported. However, the amount of molar intrusion is limited, and this approach requires an extended treatment duration. ${ }^{5,6}$ In severe cases, extraction of extruded teeth or adjunctive surgery, including corticotomy or segmental osteotomy, may be indicated. If the transverse discrepancy is beyond the range of tooth compensation, orthognathic surgery is the first option to correct 
the occlusal plane canting and posterior bite collapse in skeletal facial asymmetry.

In this case, the first option was orthognathic surgery by maxillary Le Fort-1 osteotomy for canting correction and mandible bilateral sagittal split osteotomy for facial asymmetry. However, the patient rejected orthognathic surgery. The second alternative was camouflage treatment combined with PMSO for intermaxillary space restoration (Figure 4), and the third was conventional orthodontic treatment using TADs. However, the planned amount of intrusion was more than $4 \mathrm{~mm}$, and pneumatization was observed in the maxillary sinus. Therefore, the second option was selected, and the patient was informed that skeletal asymmetry would not be eliminated without jaw surgery.

\section{TREATMENT PROGRESS}

PMSO was performed under general anesthesia. A vestibular incision was made $5 \mathrm{~mm}$ superior to the mu- cogingival junction; a horizontal bone cut was made 5 $\mathrm{mm}$ above the apices of the posterior teeth by using a 2-mm round bur; and an interdental vertical bone cut was made between the canine and first premolar (Figure 5). At this time, any detected cancellous bone was checked. The horizontal cut was finished with a curved line behind the tuberosity. Careful pressure on the maxillary segment was applied to fracture it by using a curved osteotome. After confirming segment mobilization, it was set in the correct position by using a miniplate, micromesh, and 5-mm screws. The posterior segment was impacted by $4.0 \mathrm{~mm}$ and moved $2.0 \mathrm{~mm}$ medially, guided by the wafer splint. An L-type C-tube was inserted in the alveolar bone of the PMSO site, and a miniplate (Jin Biomed Co., Bucheon, Korea) was implanted on the mandibular buccal side, distal to the second premolar area, for further orthodontic treatment.

Two weeks postoperatively, segmental bonding on the maxillary left posteriors was performed. A palatal Ctube (Jin Biomed Co.) was implanted in the mid-palatal
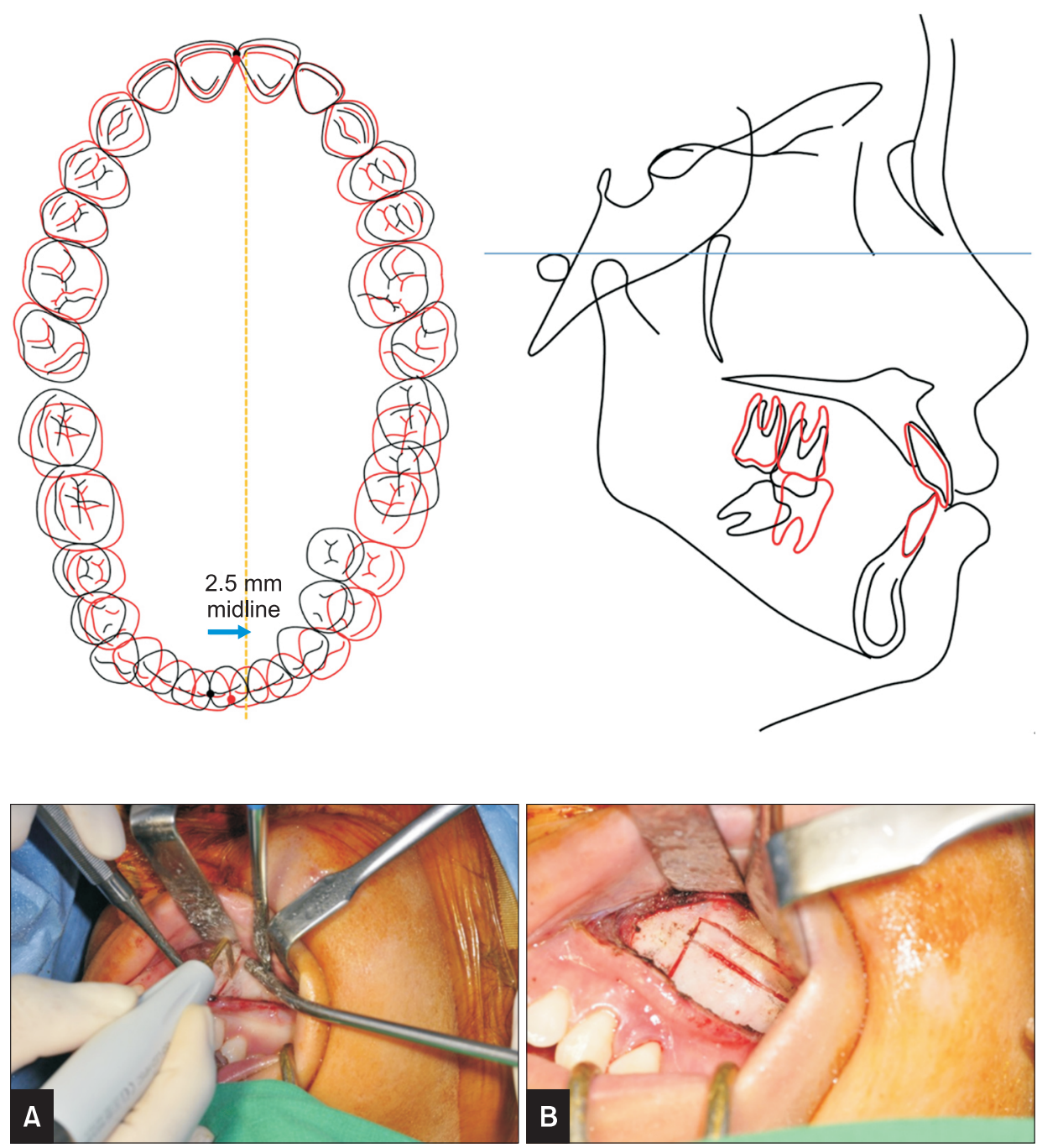

Figure 4. Cephalometric and dental visual treatment objective (VTO): the posterior teeth on the cephalometric VTO represent the collapsed side only (left). Posterior maxillary segmental osteotomy is planned on the left maxillary arch. In the mandibular arch, uprighting and protraction of the mandibular left molars and transverse compensation is planned.

Black, Pretreatment; red, VTO.

Figure 5. Posterior maxillary segmental osteotomy procedure with a vertical bone cut (A) and a horizontal bone cut (B). 
area to control the buccolingual inclination of posterior teeth. In the left mandibular arch, first and second premolars were bonded to intrude and upright buccally using the plate. A unilateral posterior bite plane on the right side, obtained using a clear overlay appliance, was delivered to facilitate uprighting of the mandibular premolars. At the same time, extrusion of mandibular left molars was achieved by using vertical elastic from the teeth to the upper C-tube (Jin Biomed Co.). After setting the vertical position of the maxillary molars, they
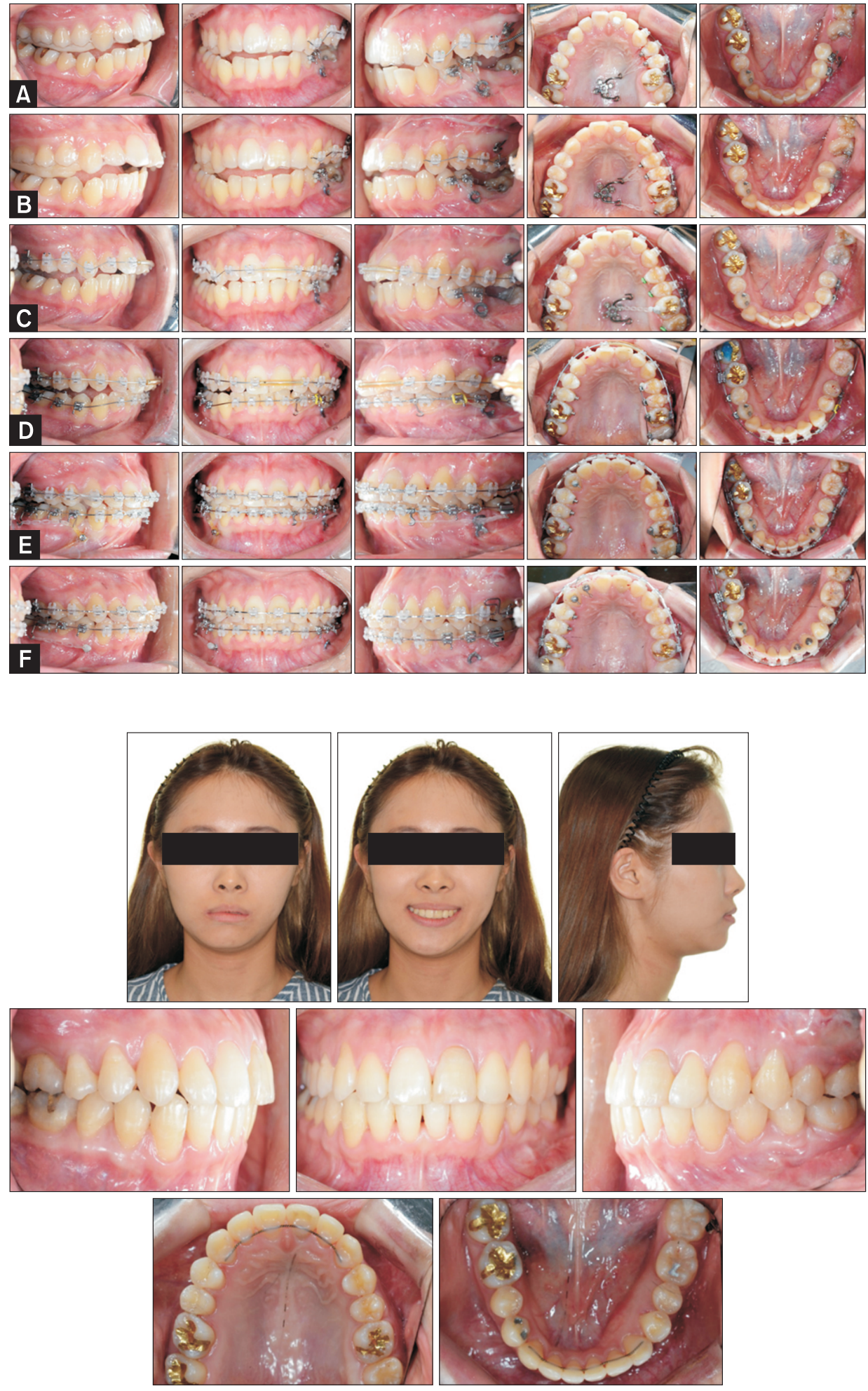

Figure 6. Treatment progression 2 weeks (A), 6 weeks (B), 10 weeks (C), 5 months (D), 11 months (E), and 16 months (F) after posterior maxillary segmental osteotomy.

Figure 7. Post-treatment facial and intraoral photographs. 
were maintained by passive ligature with the miniplate and the C-tube (Jin Biomed Co.). Three months after PMSO, full bonding on both arches was performed and leveling and alignment was started. Five months after PMSO, the mandibular left molars were erupted and engaged with a continuous arch wire. The miniplate on the left premolar area had multiple uses: protraction of the left molars, buccal uprighting and intrusion of the left premolars, and correction of the midline discrepancy. At 11 months, bonding on the left maxillary central incisors was performed after confirmation of apical lesion healing. An orthodontic mini-implant was additionally installed between the mandibular canine and first pre- molar on the right side to facilitate total arch movement to the left. The force was applied from the TAD to the right first molar with a long lever arm for bodily movement. Cross elastics and an asymmetric arch-form were applied to accomplish ideal overjet and overbite on both sides (Figure 6). The mandibular anterior teeth were stripped to correct the $1.7-\mathrm{mm}$ tooth size discrepancy.

The total treatment duration was 18 months. Immediately after debonding, wrap-around retainers and lingual bonded retainers were applied in both arches (canine to canine).
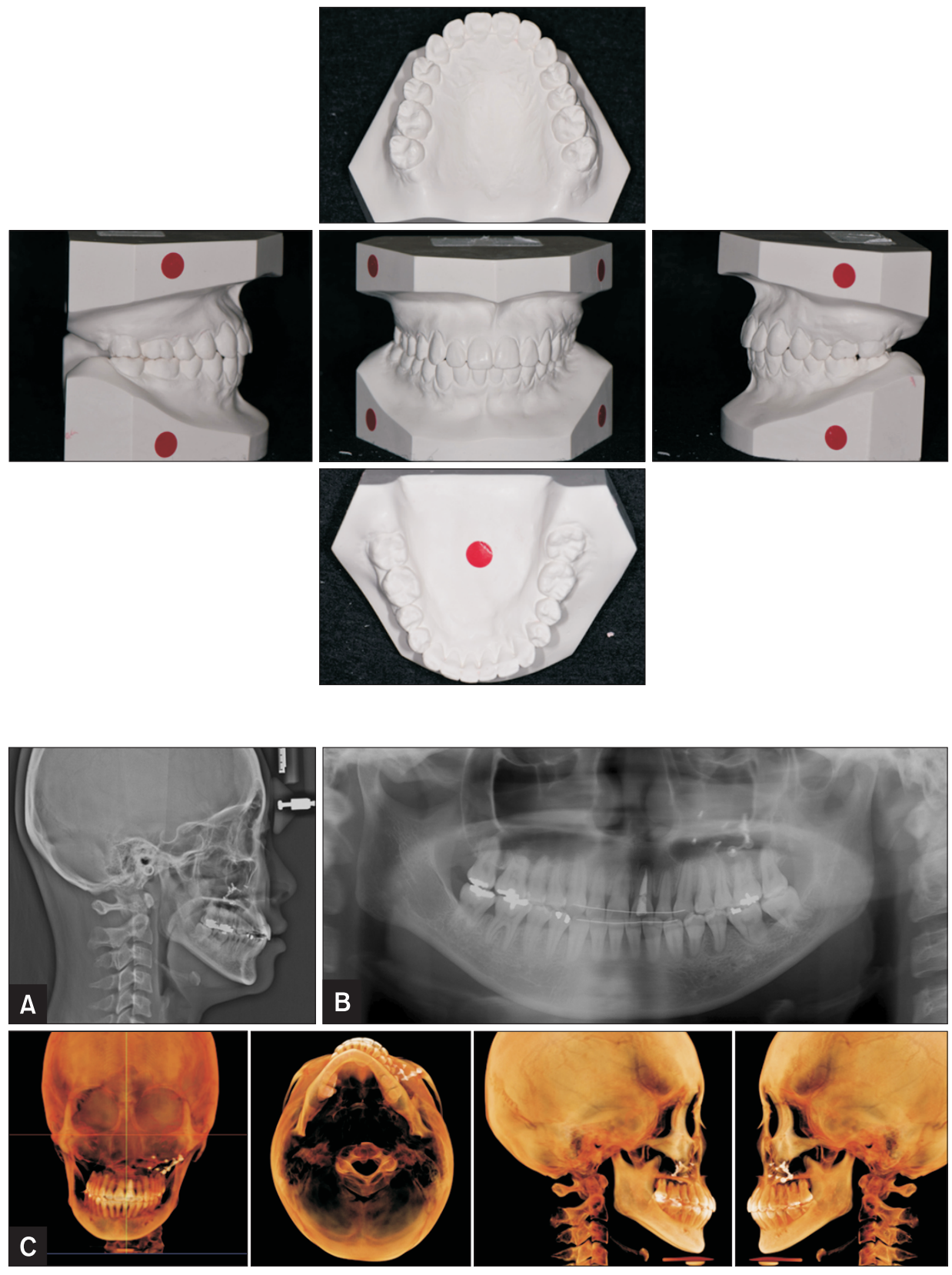

Figure 9. Post-treatment radiographs. A, Lateral cephalogram; B, panoramic radiograph; and $C_{\text {, cone-beam }}$ computed tomography images. 


\section{TREATMENT RESULTS}

The post-treatment records showed that the treatment objectives were achieved successfully. A Class 1 canine and molar relationship with ideal overbite and overjet was recorded, although there was still a small amount of lower dental midline deviation. The lateral cephalometric analysis and superimposition showed 4.0-mm intrusion of the maxillary left first molar, and $5.0-\mathrm{mm}$ protraction and 3.0-mm extrusion of the mandibular left second molar. The maxillary incisor had extruded by $1.0 \mathrm{~mm}$ while the mandibular incisors were slightly proclined and intruded by $1.0 \mathrm{~mm}$. The CBCT analysis revealed complete resolution of the occlusal plane canting ( 2 $\mathrm{mm}$ and $4 \mathrm{~mm}$ at the canine and maxillary first molar levels, respectively). PMSO helped achieve transverse and vertical dental compensation within alveolar housing. The intercanine width and intermolar width of the mandibular arch were well maintained, which contributed to long-term stability (Figures 7-11). No condylar position changes before and after treatment were observed (Figure 12).

After a 2-year retention period, the occlusion and facial profile was well maintained. The superimposition between the post-treatment and post-retention cephalograms showed stable results (Figures 13,14, and Table 1).
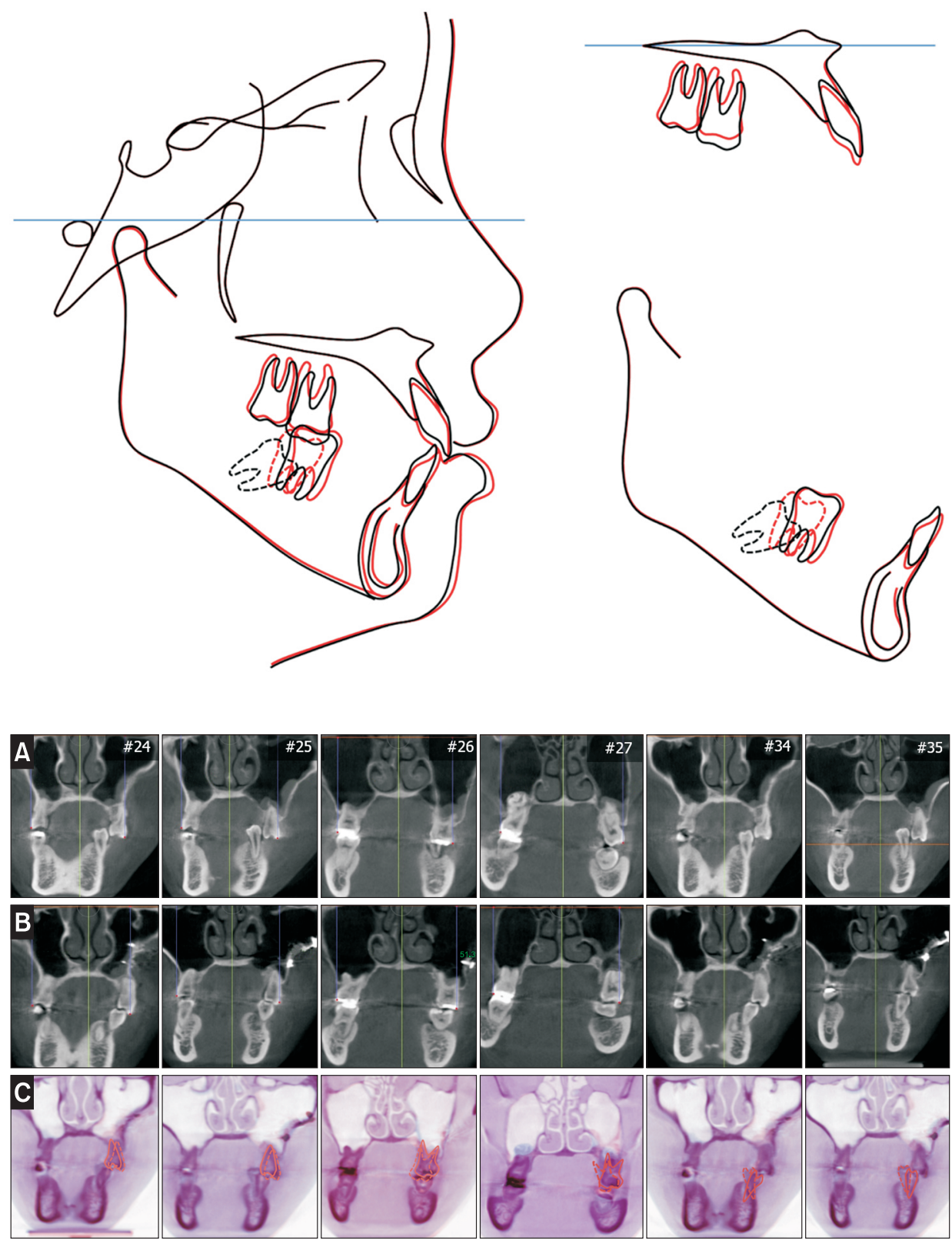

Figure 10. Cephalometric superimpositions between the pretreatment (black lines) and post-treatment stages (red lines): overall, maxilla, and mandible. For the mandibular molars, the dotted line represents the left second molar, and the solid line represents the right first molar. 


\section{KJO}
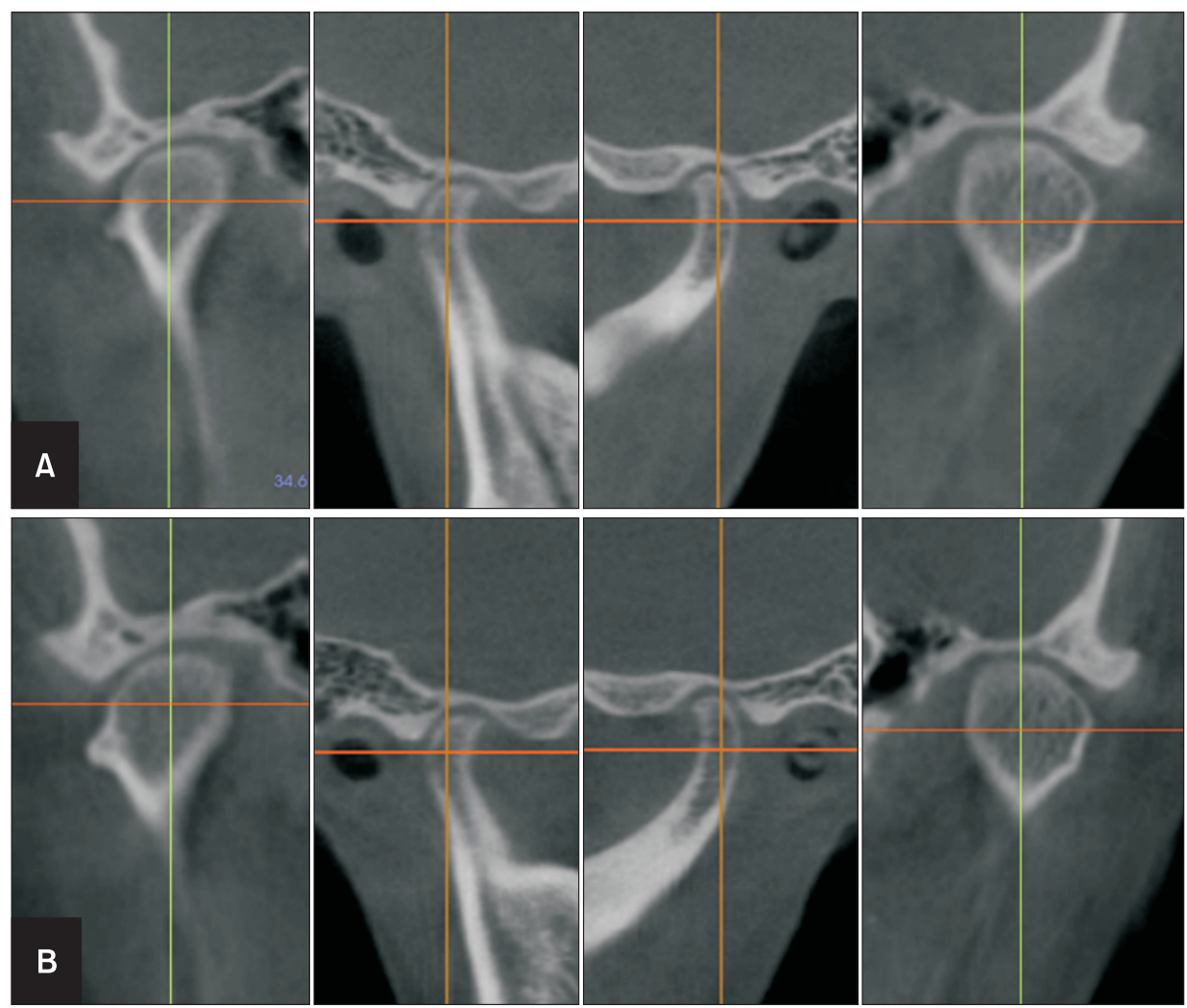

Figure 12. Temporomandibular joint position and morphology assessed using conebeam computed tomography. A, Pretreatment; B, posttreatment.

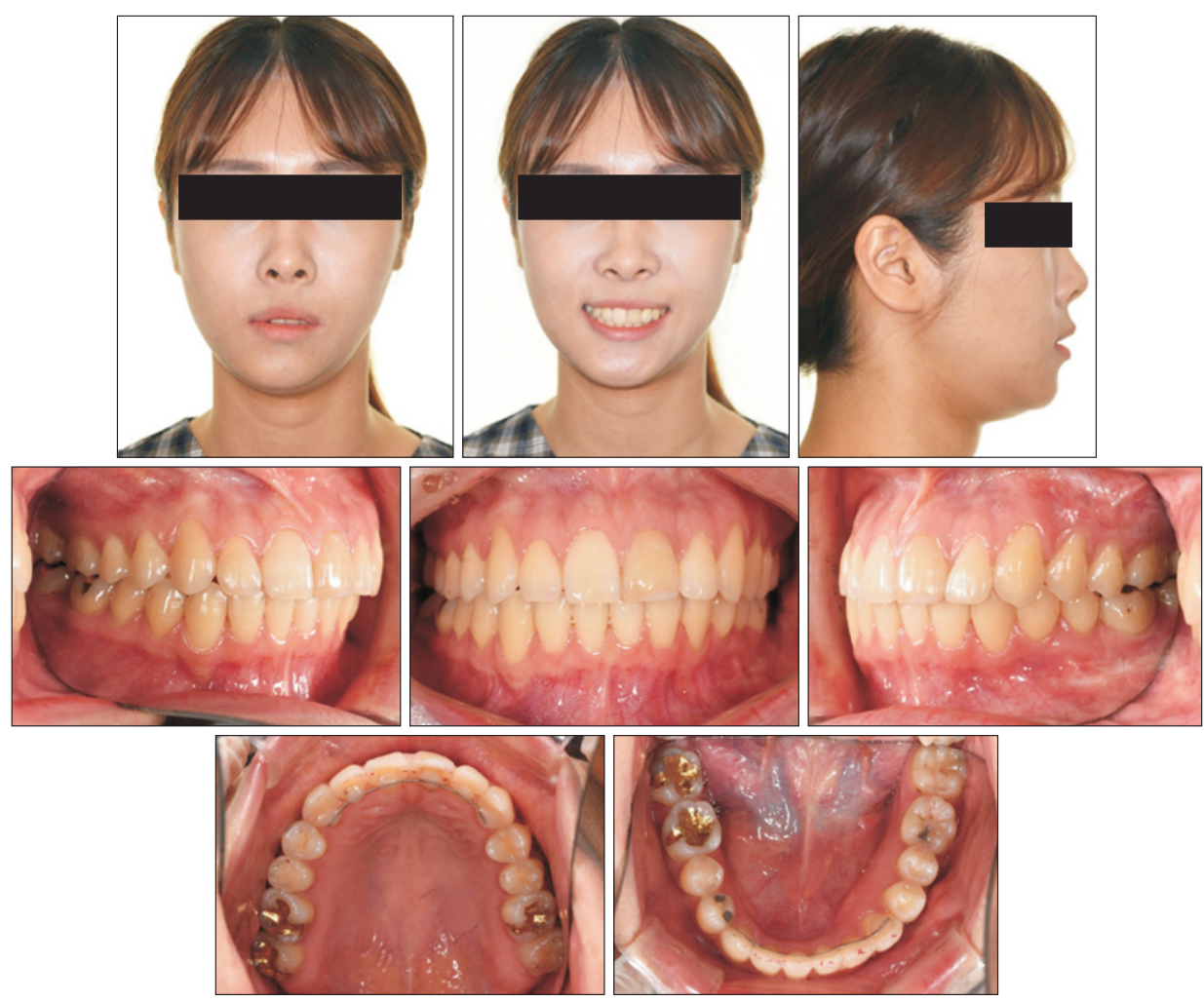

Figure 13. Post-retention (2 years) facial and intraoral photographs. 

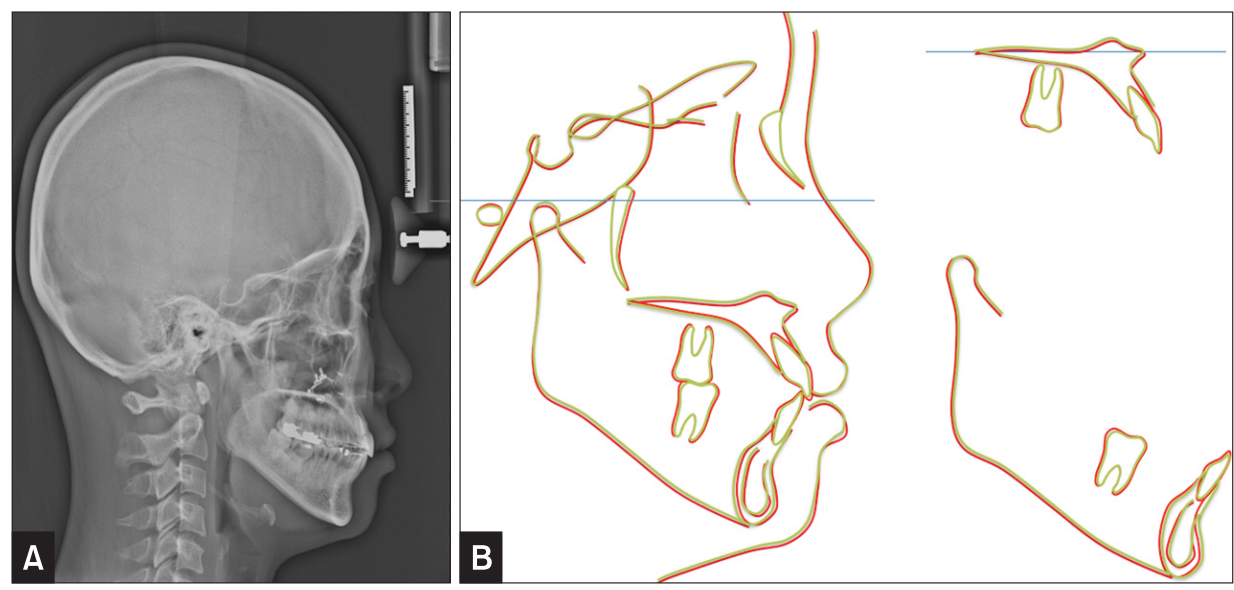

Figure 14. Lateral cephalogram at 2-year retention (A), and cephalometric superimpositions between post-treatment (red lines) and postretention stages (green lines): overall, maxilla, and mandible (B).

\section{DISCUSSION}

Dentoalveolar extrusion of posterior maxillary molars often precludes orthodontic rehabilitation of the opposing mandibular quadrant. The amount of intrusion of posterior teeth achieved by conventional orthodontic treatment is limited; however, with the recent advent of TADs, greater amounts of molar intrusion have been reported..$^{7-9}$ To resolve the occlusal plane canting, multiple methods of unilateral molar intrusion have been suggested. Kang et al. ${ }^{6}$ described the "rhythmic arch" to intrude premolars and molars together using a minimal number of TADs. Jeon et al. ${ }^{5}$ also reported that mandibular surgery was performed only in a patient with facial asymmetry and the canting was corrected using TADs. Although TADs are useful tools for intrusion of posterior teeth, their use may be limited if the amount of intrusion required is more than 3 to $4 \mathrm{~mm}$.

PMSO is an adjunctive surgery for vertical and transverse repositioning of dentoalveolar segments to correct collapsed interarch space. PMSO was developed as a two-stage surgery to correct the anterior open bite and was later modified to a one-stage procedure. ${ }^{10}$ The latter version certainly has many advantages. First, surgeons can minimize the patient's pain under general anesthesia; the treatment period is shorter; and bleeding can be controlled easily during the operation in comparison with a two-stage procedure under local anesthesia. ${ }^{11,12}$ The chief concern with this type of treatment is the potential for partial relapse, devitalization of the superiorly placed maxillary segment, and sinus complications. However, the mobilized segments remain stable because of close approximation of the bony segments, adequate interosseous fixation, and the use of a transitional opposing occlusion. ${ }^{13,14} \mathrm{Kim}$ et al. ${ }^{15}$ regained space between the maxillary first molar and mandibular alveolar crest (average, $4.6 \mathrm{~mm}$ ) with one-stage PMSO. In our case, the maxillary occlusal plane cant was resolved by $5.0-\mathrm{mm}$ intrusion of the maxillary molars on the collapsed side with PMSO. In comparison with the conventional method, TAD usage takes 5-6 months to achieve 3-4 mm of molar intrusion. ${ }^{16,17}$ PMSO shows greater advantage in shortening treatment duration and amount of intrusion.

Patients with facial asymmetry often show transverse dental compensation to maintain interarch relationship under the transverse discrepancy. ${ }^{18,19}$ Various methods have been reported to induce dental compensation for camouflage treatment in facial asymmetry patients, including asymmetric extraction, intermaxillary elastics, and torquing archwire. ${ }^{20}$ However, in complex cases, none of these approaches can overcome the anatomic limitations imposed by asymmetric arches and the thickness of the alveolar bone. If the amount of dental compensation is beyond the range of the alveolar boundary, it will be impossible to achieve ideal occlusion without surgery. PMSO is effective in intruding the segment, whereas medial or lateral movement would be relatively limited because it is difficult to secure sufficient bone contact due to the palatal shape. In our case, the transverse position of posterior teeth was varied, and the surgical extent of inward movement was determined based on the innermost first molar position. The PMSO achieved medial movement of $2 \mathrm{~mm}$, and the additional required amount was achieved by orthodontic tooth movement. The remaining maxillary posterior teeth shifted inward or were inclined palatally by using TADs after surgery as well as further transverse dental compensation of the mandibular dentition (Figure 11).

The mandibular first molar is the most frequent missing tooth. ${ }^{21} \mathrm{~A}$ thicker cortical bone with dense radially oriented trabeculae in the posterior mandibular area complicates molar protraction. The rate of mandibular molar protraction using TADs is approximately 0.34 to $0.6 \mathrm{~mm}$, which is nearly half of the maxillary molar movement rate. ${ }^{22}$ We achieved $5 \mathrm{~mm}$ molar protraction in approximately 8 months, with precise three-dimensional 
control during space closure, by using the miniplate. Molar protraction using buccal TADs along with buccal inclination of molars would be favorable with respect to the transverse compensation on the non-deviated side. Additionally, the option of extraction space closure with second molar protraction offers a periodontal advantage over prosthetic replacement in terms of bone support and oral hygiene. The second molar protraction enhances new alveolar bone formation during space closure and avoids bone damage mesial to the protracted teeth. ${ }^{23}$

The important issue related to occlusal plane canting correction is its stability. Vertical asymmetry correction by maxillary orthognathic surgery is reported to be the most stable. ${ }^{24}$ There are no long-term data supporting the stability of PMSO; however, it is expected to be more stable than conventional orthodontic treatment or corticotomy-assisted approaches, since it depends on the movement of the bony segment rather than individual tooth movement. In addition, previous studies showed that if balanced and functional occlusion was firmly established in skeletal asymmetry patients, the treatment results of camouflage treatment are well maintained. ${ }^{25,26}$ Further studies with a long follow-up period may be needed to evaluate the occlusion stability following PMSO.

\section{CONCLUSION}

The PMSO is an adjunctive and effective surgical intervention to correct posterior bite collapse in patients with facial asymmetry. The PMSO can control the posterior segment in vertical and transverse dimensions, which simplifies the orthodontic treatment and contributes to long-term stability through dental compensation within the alveolar bone housing.

\section{CONFLICTS OF INTEREST}

No potential conflict of interest relevant to this article was reported.

\section{REFERENCES}

1. Shifman A, Laufer BZ, Chweidan H. Posterior bite collapse--revisited. J Oral Rehabil 1998;25:376-85.

2. Dersot JM, Giovannoli JL. [Posterior bite collapse. 1. Etiology and diagnosis]. J Parodontol 1989;8:18794. French.

3. Sekiya T, Nakamura Y, Oikawa T, Ishii H, Hirashita A, Seto K. Elimination of transverse dental compensation is critical for treatment of patients with severe facial asymmetry. Am J Orthod Dentofacial Orthop 2010;137:552-62.
4. Kim KA, Lee JW, Park JH, Kim BH, Ahn HW, Kim SJ. Targeted presurgical decompensation in patients with yaw-dependent facial asymmetry. Korean J Orthod 2017;47:195-206.

5. Jeon YJ, Kim YH, Son WS, Hans MG. Correction of a canted occlusal plane with miniscrews in a patient with facial asymmetry. Am J Orthod Dentofacial Orthop 2006;130:244-52.

6. Kang YG, Nam JH, Park YG. Use of rhythmic wire system with miniscrews to correct occlusalplane canting. Am J Orthod Dentofacial Orthop 2010;137:540-7.

7. Daimaruya T, Takahashi 1, Nagasaka H, Umemori M, Sugawara J, Mitani H. Effects of maxillary molar intrusion on the nasal floor and tooth root using the skeletal anchorage system in dogs. Angle Orthod 2003;73:158-66.

8. Yao CC, Lee JJ, Chen HY, Chang ZC, Chang HF, Chen YJ. Maxillary molar intrusion with fixed appliances and mini-implant anchorage studied in three dimensions. Angle Orthod 2005;75:754-60.

9. Park YC, Lee SY, Kim DH, Jee SH. Intrusion of posterior teeth using mini-screw implants. Am J Orthod Dentofacial Orthop 2003;123:690-4.

10. Meningaud JP, Pitak-Arnnop P, Corcos L, Bertrand JC. Posterior maxillary segmental osteotomy for mandibular implants placement: case report. Oral Surg Oral Med Oral Pathol Oral Radiol Endod 2006;102:e1-3.

11. West RA, Epker BN. Posterior maxillary surgery its place in the treatment of dentofacial deformities. $\mathrm{J}$ Oral Surg 1972;30:562-3.

12. Bell WH, Levy BM. Revascularization and bone healing after posterior maxillary osteotomy. J Oral Surg 1971;29:313-20.

13. Rosen PS, Forman D. The role of orthognathic surgery in the treatment of severe dentoalveolar extrusion. J Am Dent Assoc 1999;130:1619-22.

14. O'Ryan F, Schendel S. Nasal anatomy and maxillary surgery. 11. Unfavorable nasolabial esthetics following the Le Fort 1 osteotomy. Int J Adult Orthodon Orthognath Surg 1989;4:75-84.

15. Kim C, Kim M, Kim D. Upward repositioning of the extruded posterior maxillary alveolar segment by one-stage segmental osteotomies. Paper presented at: 14th International Conference on Oral and Maxillofacial Surgery; 1999 Apr 24-29; Washington DC, USA. Chicago: ICOMS, 1999. p. 13

16. Sherwood KH, Burch J, Thompson W. Intrusion of supererupted molars with titanium miniplate anchorage. Angle Orthod 2003;73:597-601.

17. Erverdi N, Keles A, Nanda R. The use of skeletal anchorage in open bite treatment: a cephalometric evaluation. Angle Orthod 2004;74:381-90. 
18. Kusayama M, Motohashi N, Kuroda T. Relationship between transverse dental anomalies and skeletal asymmetry. Am J Orthod Dentofacial Orthop 2003;123:329-37.

19. Solow B. The dentoalveolar compensatory mechanism: background and clinical implications. Br J Orthod 1980;7:145-61.

20. Anhoury PS. Nonsurgical treatment of an adult with mandibular asymmetry and unilateral posterior crossbite. Am J Orthod Dentofacial Orthop 2009;135:118-26.

21. Meskin LH, Brown LJ. Prevalence and patterns of tooth loss in U.S. employed adult and senior populations, 1985-86. J Dent Educ 1988;52:686-91.

22. Roberts WE, Arbuckle GR, Analoui M. Rate of mesial translation of mandibular molars using implantanchored mechanics. Angle Orthod 1996;66:331-8.
23. Raveli TB, Raveli DB, de Mathias Almeida KC, Pinto ADS. Molar uprighting: a considerable and safe decision to avoid prosthetic treatment. Open Dent J 2017;11:466-75.

24. Proffit WR, Turvey TA, Phillips C. The hierarchy of stability and predictability in orthognathic surgery with rigid fixation: an update and extension. Head Face Med 2007;3:21.

25. Jung SK, Kim TW. Treatment of unilateral posterior crossbite with facial asymmetry in a female patient with transverse discrepancy. Am J Orthod Dentofacial Orthop 2015;148:154-64.

26. Kai R, Umeki D, Sekiya T, Nakamura Y. Defining the location of the dental midline is critical for oral esthetics in camouflage orthodontic treatment of facial asymmetry. Am J Orthod Dentofacial Orthop 2016;150:1028-38. 